\title{
Head-to-head comparison of pre-hospital qSOFA and lactate-qSOFA for predicting sepsis in patients with and without suspected infection. A multicenter prospective cohort study
}

\author{
Francisco Martín-Rodríguez ${ }^{1,2}$, Raúl López-Izquierdo ${ }^{3}$, Miguel A. Castro Villamor ${ }^{1}$, \\ Carlos delPozo-Vegas ${ }^{4}$, Irene Sánchez-Soberón², Juan F. Delgado-Benito², \\ Virginia Carbajosa Rodíguez ${ }^{3}$, Rodrigues Leonardo ${ }^{5}$, José Luis Martín-Conty ${ }^{6}$
}

\author{
${ }^{1}$ Advanced Clinical Simulation Center, Faculty of Medicine, Universidad de Valladolid, \\ Valladolid, Spain \\ ${ }^{2}$ Emergency Medical Services, Gerencia de Emergencias Sanitarias de Castilla y León, \\ Valladolid, Spain \\ ${ }^{3}$ Emergency Department, Hospital Universitario Rio Horteg, Valladolid, Spain \\ ${ }^{4}$ Emergency Department, Hospital Clínico Universitario, Valladolid, Spain \\ ${ }^{5}$ Surgery Department, Hospital Sanatorio de Luanda, Angola, Spain \\ ${ }^{6}$ Faculty of Health Sciences, Universidad de Castilla la Mancha, Talavera de la Reina, \\ Spain
}

Submitted: 30 November 2019

Accepted: 18 January 2020

Arch Med Sci

DOI: https://doi.org/10.5114/aoms.2020.92901

Copyright $\odot 2020$ Termedia \& Banach

\begin{abstract}
Introduction: The aim of the study was to compare the Quick Sequential Organ Failure Assessment tools (qSOFA), and Quick Sequential Organ Failure Assessment-Lactate (LqSOFA) to detect pre-hospital sepsis among patients with or without suspicion of infection.

Material and methods: Multicenter prospective cohort study in non-traumatic patients attended by Advanced Life Support in the prehospital environment and transferred to the hospital. We collected demographic, physiological, clinical, and analytical variables, main diagnosis, hospital admission and clinical diagnosis of sepsis. Primary outcome was the clinical diagnosis of sepsis in the hospital.

Results: Between March 1, 2018 and March 31, 2019, we included in our study 1548 participants of whom 82 (5.3\%) received a final hospital diagnosis of sepsis. The qSOFA presented an area under the receiver operating characteristic curve (AUROC) to detect sepsis in patients with suspicion and without suspicion of infection of 0.757 (95\% Cl: $0.65-0.81)$, compared to LqSOFA with an AUROC to detect sepsis of $0.784(95 \% \mathrm{Cl}: 0.72-0.84)$. In the group of patients with suspected infection, the LqSOFA for a 2-point cut-off presented an AUROC of 0.773 (95\% Cl: $0.69-0.85)$, with an excellent sensitivity of $92.6 \%$ (95\% Cl: $83.9-96.8)$ and an odds ratio of 8.23 (95\% Cl: $3.09-21.92$ ). Conclusions: An appropriate strategy for reducing the morbidity and mortality from sepsis must necessarily include the prompt identification of this time-dependent pathology by using all the tools at our disposal. The qSOFA and LqSOFA can be used in the prehospital environment and help the diagnosis of suspected sepsis in patients with medical pathology, highlighting the predictive capacity of LqSOFA in the group of patients with suspected infection.
\end{abstract}

Key words: prehospital care, sepsis, point-of-care testing, warning score, clinical decision.
Corresponding author: Raúl López-Izquierdo MD, $\mathrm{PhD}$ Emergency Department Rio Hortega University Hospital of Valladolid SACYL. C/Dulzaina 2 47012 Valladolid, Spain Phone: 646557285 E-mail: rlopeziz@ saludcastillayleon.es 
F. Martín-Rodríguez, R. López-Izquierdo, M.A. Castro Villamor, C. delPozo-Vegas, I. Sánchez-Soberón, J.F. Delgado-Benito, V. Carbajosa Rodíguez, R. Leonardo, J.L. Martín-Conty

\section{Introduction}

Sepsis is a serious disease whose early identification is directly related to survival $[1,2]$. Emergency medical services (EMS) are challenged by the detection of this time-dependent pathology $[3,4]$. However, rapid recognition of the situation guides the appropriate life support measures for this heterogeneous and complex condition $[5,6]$.

With the latest consensus definition of sepsis and septic shock, the quick Sequential Organ Failure Assessment (qSOFA) has been advocated as a tool for the early detection of suspected sepsis outside the intensive care units (ICU) $[1,7]$. qSOFA is performed by determining three simple clinical parameters (systolic blood pressure below or equal to $100 \mathrm{~mm} \mathrm{Hg}$, respiratory rate above $22 \mathrm{bpm}$, and altered mental status). A qSOFA of 2 points directs us towards a diagnosis, and the implementation of appropriate measures [8].

Point-of-care lactate has been used routinely as a reliable standard indicator of anaerobic metabolism, and therefore, of tissue hypoperfusion in sepsis $[1,9]$. In the prehospital context, prehospital lactate ( $p L A)$ is measured if sepsis is suspected $[10,11]$. A pLA greater than or equal to $2 \mathrm{mmol} / \mathrm{l}$ and suspicion of infection suggests close monitoring and control of lactate values [12]. Values above $4 \mathrm{mmol} / \mathrm{l}$ activate the prehospital sepsis code [13].

The impact of prehospital care on sepsis is still to be determined, but it is beyond doubt that the early diagnosis of suspected sepsis improves the prognosis [14, 15]. The concept of the "prehospital golden hour" in sepsis should be used routinely by EMS because rapid identification, aggressive volume management (if necessary), determination of the pLA, rapid evacuation and hospital pre-notification should be the basis for the initial management of the patient with suspected sepsis $[13,16]$.

In the prehospital setting, where diagnostic procedures are limited, any help should be evaluated. The use of specific scales such as the qSOFA or point-of-care testing as the pLA, or mixed tools such as the quick Sequential Organ Failure Assessment with lactate (LqSOFA), for the detection of sepsis, represent a fundamental aid for professionals.

Although many studies have evaluated the use of qSOFA and lactate (LqSOFA) as a predictor of mortality, not enough prospective studies in the prehospital setting have assessed their usefulness for the early detection of sepsis (and not just mortality).

The objective of this study was to compare different sepsis detection tools at the prehospital level (qSOFA and LqSOFA) to determine the presence of sepsis (hospital clinical diagnosis), both globally and in the case of suspected infection [1].

\section{Material and methods}

\section{Study design and setting}

We conducted a multicenter prospective cohort study between March 1, 2018 and March 31, 2019 with seven advanced life support units (ALS) and five hospitals. We included all non-traumatic patients who were attended by ALS and transferred to the hospitals in the provinces of Burgos, Salamanca, Segovia and Valladolid (Spain), with a reference population of 1,113,073 inhabitants.

The EMS is integrated by a call center (1-1-2), which operates $24 \mathrm{~h}$ a day, 365 days a year. The EMS depends on the public health system of the Community of Castilla y León (Spain) and is composed of $23 \mathrm{ALS}$, and 117 basic life support units (BLS). The emergency call is analyzed by an emergency registered nurse (ERN) and/or medical doctor (MD), who, upon evaluation, send the most appropriate resource to the incident site. The ALS is composed of two paramedics and an ERN and an MD. The staff have received specific training in advanced life support, advanced trauma training, disaster management, care for pregnant women and childbirth and management of social and psychiatric problems. The EMS operates by protocols, performing advanced life support maneuvers at the incident site or en route.

The study included admissions in four tertiary university hospitals, and one small general district hospital. The entry point of all patients was the emergency department (ED). All hospitals have an ample surgical capacity, an ICU and post-surgical resuscitation unit. All of them belonging to the public health system of the Community of Castilla y León (Spain).

The selection of hospitals was made based on the fact that in the same city there was a reference ALS. No regional hospitals were chosen or those without sufficient capacity to solve complex pathologies, where in many cases it is necessary to carry out evacuations to high-level hospitals.

\section{Population}

The sample was recruited from among all calls for urgent assistance that were received in the call center under 1-1-2. To be considered suitable for the study, patients had to exceed 18 years of age and be treated for pathology of medical origin and transferred to the hospital by an ALS.

Exclusion criteria were trauma (deliberate selfharm and overdose were also excluded), pregnancy, acute psychiatric pathology, terminal state, and cardiorespiratory arrest. We also excluded patients who were evacuated by other means of transport or discharged in situ, patients for whom follow-up through electronic medical records was not possible (when the patient does not have 
a Public Health System card, follow-up is not possible), patients who did not give informed consent and patients who were attended more than once, considering only the first chronological event for the study.

\section{Outcomes}

The primary outcome was the clinical diagnosis of sepsis at the hospital level.

In addition, we analyzed the performance of these scales for the subgroups of patients with suspected infection and without suspicion of infection (at the prehospital level).

For the clinical diagnosis of sepsis, an associated investigator from each hospital performed the SOFA score, in line with other publications [1, $6,8]$. For the present study, there was no difference between sepsis and septic shock; both cases were considered as a clinical diagnosis of sepsis. The criteria to consider that a patient presented sepsis were suspected infection and a SOFA score greater than or equal to two points (starting from a baseline SOFA of 0 ) or increase of two points with respect to the baseline SOFA score (e.g. a patient with renal insufficiency and part with a baseline score that is not zero).

The principal investigator made periodic visits (once a month) to each ALS and ED of each hospital, to answer questions and verify the correct collection of the study variables. The principal investigator has reviewed the data of $50 \%$ of the sample and of the total cases of diagnosis of sepsis.

\section{Methods of measurement}

All patient data were recorded electronically, in a database created for this purpose, where the MD of the ALS introduced the demographic variables (sex and age), times of arrival, assistance and evacuation, advanced life support maneuvers of special follow-up (use of supplementary oxygen, advanced management of the airway - orotracheal intubation, non-invasive ventilation and difficult airway - and use of intravenous medication), suspicion of infection and prehospital diagnosis.

The parameters necessary to calculate the qSOFA [8] (systolic blood pressure, respiratory rate and mental state) and the venous blood sample for the determination of the pLA were collected by the ERN at the first contact with the patient during prehospital care. Systolic blood pressure was measured with the LifePAK 15 monitor (Physio-Control, Inc., Redmond, USA). Respiratory rate was measured by counting for 1 min the number of complete respiratory cycles (inspiration and expiration) performed by the participant, by direct observation or in case of doubt by auscultation. The mental state was assessed using the Glasgow
Coma Scale (confusion was defined as a Glasgow Coma Scale score of less than 15 points).

To obtain PLA values, the Accutrend Plus measuring device (Roche Diagnostics, Mannheim, Germany) was used, with a measuring range of 0.8-21.7 mmol/l. The entire procedure consists of three phases: first, the instrument is switched on and the test strip is inserted; second, a drop of blood from the venous line (extracted in a $1 \mathrm{ml}$ syringe) is deposited on the test strip $(15-40 \mu l)$; and third, the lid closes and a result is obtained after $60 \mathrm{~s}$. Between the extraction of blood and the placement of the sample in the device, no more than 1 min should pass. All measuring devices were calibrated every 50 measurements, always by the same researcher, by means of a control solution, Accutrend BM-Control-Lactate (Roche Diagnostics, Mannheim, Germany).

The remaining data were obtained through review of the electronic patient records 30 days after the index event by an associated researcher of each hospital: hospital diagnosis of sepsis [1] and infectious focus, need for hospitalization and/or ICU, days of hospitalization and/or ICU and mortality in the hospital from sepsis.

\section{Calculation of LqSOFA}

To calculate the LqSOFA, the numerical value of the pLA was converted into an ordinal variable. For this, we established three groups of pLA. PLA values up to the $25^{\text {th }}$ percentile were assigned 0 points, values between the $25^{\text {th }}$ and $75^{\text {th }}$ percentiles were assigned 1 point, and pLA values above the $75^{\text {th }}$ percentile were assigned 2 points. The cut-off point for the $25^{\text {th }}$ percentile is $2.1 \mathrm{mmol} / \mathrm{l}$, a value consistent with the clinical data suggesting the presence of sepsis [17]. The cut-off point for the $75^{\text {th }}$ percentile is $4.2 \mathrm{mmol} / \mathrm{l}$, which indicates the presence of frank lactic acidosis [18, 19]. The score obtained by converting the quantitative value of the pLA into a qualitative value is added to the qSOFA score, and thus, a single LqSOFA scale is obtained that is supported by clinical and statistical criteria.

Throughout the article, when referring to the pLA, we refer to the qualitative lactate value.

\section{Primary data analysis}

Before statistical analysis, the database was cleaned using logical tests, range tests (for the detection of extreme values) and tests for consistency of the data. Subsequently, we analyzed the presence and distribution of unknown values of all the variables.

All data were stored in an XLSTAT BioMED (version 2019.3.2) database for Microsoft Excel (version 14.4.0). Data were analyzed with IBM SPSS 
Statistics, version 20.0. The data are presented according to the Standards for Reporting Diagnostic Accuracy 2015 statement [20].

We calculated the area under the curve (AUC) of the receiver-operating characteristic (ROC) for each of the scales qSOFA and LqSOFA, both in patients with suspected prehospital infection and in patients without suspected infection. We determined the cut-off point of each scale that offered the highest sensitivity and specificity using the Youden index, calculating in each case sensitivity, specificity, positive predictive value, negative predictive value, likelihood ratio, odds ratio and diagnostic accuracy. By means of nonparametric contrasts, the equality of the AUC obtained was tested.

\section{Statement of human rights}

The study was approved by the Research Ethics Committee of all participating centers (reference: \#PI 18-010, \#PI 18-895, \#PI 18-10/119, \#PI MBCA/dgc and \#PI CEIC 2049). All patients (or guardians) signed informed consent. The study was carried out with the highest safety standards, protecting the physical integrity and confidentiali-

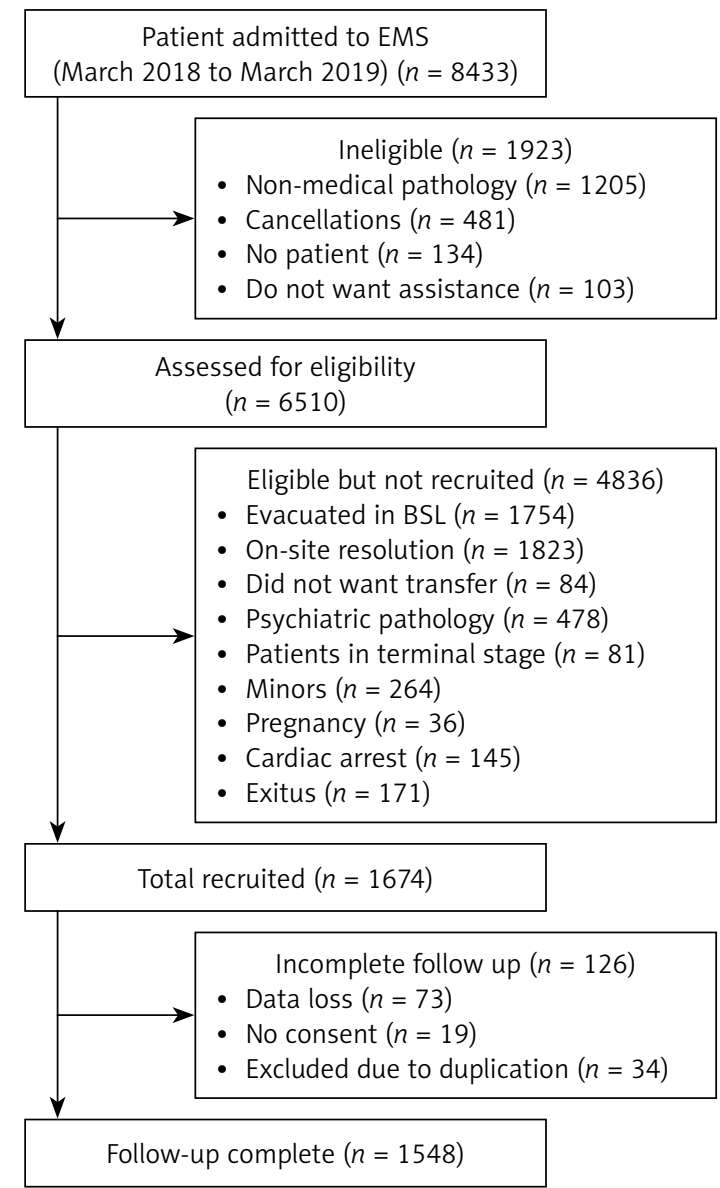

Figure 1. Flowchart of participants in the study EMS-emergency medical service, BLS - basic life support. ty of the participants, complying with national and international regulations for the study of human subjects included in the Declaration of Helsinki.

The study protocol is available online (doi. org/10.1186/ISRCTN17676798); we follow the STROBE statement for reporting.

Informed consent was obtained from all individual participants included in the study (in the case that the patient due to (ITS seriousness) could not grant consent, a family member or tutor was spoken to, and in the latter case in the subsequent hospital follow-up informed consent was obtained).

\section{Results}

\section{Characteristics of study subjects}

Between March 1, 2018 and March 31, 2019, a total of 1548 participants with acute non-traumatic disease were included in our study (Figure 1), of whom 82 (5.3\%) had sepsis confirmed during their emergency department evaluation. Median age was 72 years (IQR: $59-83$ years) and 612 (39.5\%) were women.

We systematically evaluated respiratory rate, systolic blood pressure, Glasgow Coma Scale score and quantitative lactate which was later qualitatively recoded and found statistically significant differences in all analyzed values between patients with sepsis and without sepsis $(p=0.001)$ (Table I).

In patients with sepsis, $75.6 \%$ required supplemental oxygen, $20.7 \%$ had advanced airway management, and $89.0 \%$ received intravenous medication. $31.4 \%$ of patients without diagnosed sepsis needed supplemental oxygen, 5.0\% advanced airway management and $75.2 \%$ received intravenous medication (in all cases $p<0.05)$ (Table I).

In 192 (12.4\%) cases, infection was suspected at the prehospital level. Overall, 82 (5.3\%) patients were diagnosed with sepsis in the hospital, of whom 26 (31.7\%) patients died in less than 48 h, 36 (43.9\%) subjects within 7 days and $52(63.4 \%)$ within 30 days. Of the patients with sepsis, 28 (34.1\%) required ICU. The mortality of these patients was associated with age and male gender (in both cases $p<0.05$ ).

The most common infectious focus was of respiratory origin $(60.9 \%)$ with 50 cases, followed by foci of urinary origin (19.5\%), undetermined (9.7\%), abdominal (6.0\%) and neurological (3.6\%).

The prevalence of hospital admission of the total sample was 58.2\% (901 patients), with a median hospital stay of 7 days (IQR: 1-16 days) in patients with sepsis compared to 2 days (IQR: $0-7$ ) in patients not diagnosed with sepsis $(p<0.001)$. Of the patients diagnosed with sepsis, 28 (34.1\%) required admission to the ICU $(p=0.003)$ (Table I). 


\section{Main results}

The predictive power of the qSOFA scale to discriminate the presence of sepsis was evidenced by an AUC of the ROC curve of $0.757(95 \% \mathrm{Cl}$ : $0.65-0.81)$ and LqSOFA reached 0.784 (95\% Cl: $0.72-0.84)(p<0.001$ for all the scales studied) (Figure 2). Comparing the scales, no significant differences were observed between the LqSOFA scale and the qSOFA $(p=0.070)$ (Table II). Of all cases of sepsis confirmed at the hospital level, in 28 (34.1\%) cases there was no prehospital suspicion of infection (Table I).

The highest performance score for the early detection of sepsis in the group of patients with prehospital suspicion of infection is LqSOFA, with an AUC 0.773 ( $95 \% \mathrm{Cl}$ : 0.69-0.85), compared to qSOFA with an AUC of 0.673 (95\% Cl: 0.58-0.76) ( $p<$ 0.001 for the two scores studied). The comparison of the curves was significant for the LqSOFA with

Table I. General patient characteristics (statistics rates refer to clinical diagnosis of sepsis)

\begin{tabular}{|c|c|c|c|c|}
\hline Parameter & Total & Non-sepsis & Sepsis & $P$-value \\
\hline Number, $n(\%)$ & $1548(100)$ & $1466(94.7)$ & $82(5.3)$ & \\
\hline Age [years old] [median (IQR)] & $72(59-83)$ & $72(58-83)$ & $79(71-85)$ & $<0.001$ \\
\hline \multicolumn{5}{|l|}{ Sex, $n(\%):$} \\
\hline Male & $936(60.5)$ & $873(59.5)$ & $63(76.8)$ & \\
\hline Female & $612(39.5)$ & $593(40.5)$ & $19(23.2)$ & 0.001 \\
\hline \multicolumn{5}{|l|}{ Isochronous, median (IQR): } \\
\hline Arrival time & $10(8-13)$ & $10(8-13)$ & $10(8-14)$ & 0.818 \\
\hline Support time & $28(23-34)$ & $28(23-34)$ & $31(25-38)$ & 0.246 \\
\hline Transfer time & $10(7-14)$ & $10(7-13)$ & $10(5-15)$ & 0.792 \\
\hline \multicolumn{5}{|l|}{ Initial evaluation, median (IQR): } \\
\hline $\mathrm{BR}[\mathrm{bpm}]$ & $18(14-24)$ & $17(14-23)$ & $30(18-38)$ & 0.001 \\
\hline $\mathrm{SBP}[\mathrm{mm} \mathrm{Hg}]$ & $139(119-158)$ & $139(120-159)$ & $135(96-147)$ & 0.001 \\
\hline GCS [points] & $15(15-15)$ & $15(15-15)$ & $15(11-15)$ & 0.001 \\
\hline \multicolumn{5}{|l|}{ Sepsis scores, median (IQR): } \\
\hline qSOFA [points] & $1(1-1)$ & $0(0-1)$ & $1(1-2)$ & 0.001 \\
\hline $\mathrm{pLA}[\mathrm{mmol} / \mathrm{l}]$ & $2.9(2.1-4.2)$ & $2.9(2.0-4.1)$ & $4.5(3.0-6.2)$ & 0.001 \\
\hline pLA qualitative [points] & $1(0-1)$ & $1(0-1)$ & $2(1-2)$ & $<0.001$ \\
\hline LqSOFA [points] & $1(1-2)$ & $1(1-2)$ & $3(2-4)$ & 0.001 \\
\hline \multicolumn{5}{|l|}{ Prehospital support, $n$ (\%): } \\
\hline Supplemental oxygen & $522(33.7)$ & $460(31.4)$ & $62(75.6)$ & $<0.001$ \\
\hline Advanced airway & $90(5.8)$ & $73(5.0)$ & $17(20.7)$ & 0.001 \\
\hline Intravenous medication & $1176(76.0)$ & $1103(75.2)$ & $73(89.0)$ & 0.004 \\
\hline \multicolumn{5}{|l|}{ Prehospital diagnosis, $n$ (\%): } \\
\hline Non-infection suspicion & $1356(87.6)$ & $1328(97.9)$ & $28(2.1)$ & \\
\hline Infection suspicion & $192(12.4)$ & $138(71.9)$ & $54(28.1)$ & 0.001 \\
\hline \multicolumn{5}{|l|}{ Hospital care: } \\
\hline Inpatients, $n(\%)$ : & $901(58.2)$ & $819(55.9)$ & $82(100)$ & 0.001 \\
\hline ICU, $n(\%):$ & $285(18.4)$ & $257(17.5)$ & $28(34.1)$ & 0.003 \\
\hline $\begin{array}{l}\text { Hospitalization time, median (IQR) } \\
\text { [days] }\end{array}$ & $2(0-7)$ & $2(0-7)$ & $7(1-16)$ & $<0.001$ \\
\hline
\end{tabular}

Values expressed as total number (fraction) and medians [25 th percentile- $75^{\text {th }}$ percentile] as appropriate. IQR - interquartile range, $B R$ - breathing rate, SBP - systolic blood pressure, GCS - Glasgow Coma Scale, qSOFA - quick Sequential Organ Failure Assessment, pLA - prehospital lactate, LqSOFA - Lactate quick Sequential Organ Failure Assessment, ICU - intensive care unit. 


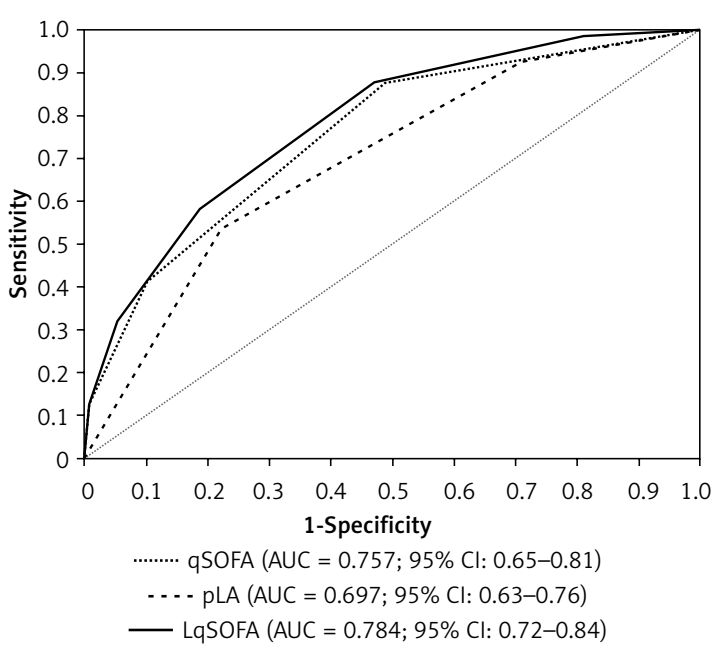

Figure 2. Diagnostic performance curves and areas under the curve with $95 \%$ confidence intervals for qSOFA, pLA and LqSOFA for clinical diagnosis of sepsis $A U C$ - area under the curve; $\mathrm{Cl}$ - confidence interval, qSOFA - quick Sequential Organ Failure Assessment, pLA - prehospital lactate, LaSOFA - Lactate quick Sequential Organ Failure Assessment, $\mathrm{Cl}$ - confidence interval.

respect to the qSOFA ( $p=0.001)$. For a 2 -point cut-off, the LqSOFA has a sensitivity of $90.7 \%$ (95\% Cl: 80.1-96.0), with a negative predictive value of $92.6 \%$ (95\% Cl: 83.9-96.8) and an odds ratio of 8.23 (95\% Cl: 3.09-21.92). These data corroborate the best cut-off points of the scales in terms of sensitivity, specificity, predictive values, likelihood ratios and unadjusted odds ratio (Table II).

In the group of patients without suspected infection (at the prehospital level), both qSOFA and LqSOFA obtained an AUC of $0.750(95 \% \mathrm{Cl}$ : $0.64-0.85$ ) (in both cases $p<0.001$ ). The comparison of the curves was not significant ( $p=0.984$ ). The qSOFA presented a sensitivity of $89.3 \%$ (95\% Cl: 72.8-96.3), with a negative predictive value of $99.6 \%(95 \% \mathrm{Cl}$ : $98.8-99.9)$ and an odds ratio of 9.49 (95\% Cl: 2.85-31.58) (Table II).

Table III shows that high scores on both qSOFA and LqSOFA scales were significantly associated with having sepsis. Additionally, $12.2 \%$ of all patients with a sepsis diagnosis presented a score on the LqSOFA scale of less than 2, while this value rose to $58.5 \%$ among patients with a qSOFA less than 2 points. Higher sepsis rates increase as the score of both qSOFA and LqSOFA increases, in both patients with suspected infection and those in whom there was no prehospital suspicion of infection.

\section{Discussion}

In this multicenter observational cohort study in the prehospital setting, we evaluated the capacity of a scale, called LqSOFA, to identify suspected sepsis. This is the first research that evaluates the joint performance of the qSOFA and the LqSOFA at prehospital level to detect patients at risk of developing sepsis. A qSOFA equal to or greater than 1 point or LqSOFA equal to or greater than 2 points serves to identify patients at high risk of presenting sepsis. As reflected in the results, this cut-off point presents high sensitivity while maintaining a powerful negative predictive value.

Both scales can be used, but the LqSOFA by including the value of lactate aligns with the international recommendations for sepsis management and can provide very useful information about the patient's perfusion status [1, 12, 13].

In the group of patients with suspected infection, the LqSOFA presented excellent sensitivity, well above that obtained in the group of patients without suspected infection, which makes the LqSOFA score a very good tool for detecting sepsis in patients with suspected infection, and with better data than qSOFA $[2,16]$.

Our results confirm and improve the data presented in other studies that evaluate the predictive capacity of qSOFA together with lactate with respect to mortality. Yet, all the studies were performed at the hospital level and none specifically analyzed the capacity of the scales studied to predict the presence of sepsis from a clinical point of view [21-25].

Sepsis should be considered a syndrome (or set of syndromes) with very heterogeneous presentations and with diverse signs and symptoms, involving inflammatory, immunological and hematological processes. An appropriate strategy for reducing morbidity and mortality due to sepsis must necessarily include the identification of this time-dependent pathology as soon as possible by using all the tools at our disposal $[12,13]$.

The third sepsis consensus [1] advocates the use of qSOFA as a tool for identifying patients at high risk of sepsis outside the ICU [26], and simultaneous point-of-care testing with biomarkers such as the pLA that can guide the diagnostic process [10, 11]. No biomarker or scale, however, has proven to be the gold standard for the early detection of sepsis [27].

A high score in the qSOFA is associated with high mortality [7], but these patients already present an evidently poor clinical situation. The combined use of qSOFA and pLA (LqSOFA) may help to discriminate patients in a severe condition who present less evident signs and symptoms, but with metabolic signs of hypoperfusion that the pLA can identify. In this way, patients at risk of sepsis, who may otherwise go unnoticed, can be assessed early [22].

As mentioned, the LqSOFA scale has good sensitivity and combined specificity for the clinical diagnosis of sepsis. The best cutoff point for suspecting sepsis in patients of the group with suspected infection from a clinical point of view 
Table II. Statistics rates refer to clinical diagnosis of sepsis for LqSOFA and qSOFA, globally and in patients with suspicion of prehospital infection and non-infection suspicion

\begin{tabular}{|c|c|c|}
\hline Parameter & qSOFA & LqSOFA \\
\hline \multicolumn{3}{|c|}{ All patients $(n=1548$, sepsis $=82)$ : } \\
\hline Cut-off [points] & 1 & 2 \\
\hline Se \% $[95 \% \mathrm{Cl}]$ & $87.8(79.0-93.2)$ & $87.8(79.0-93.2)$ \\
\hline Sp \% [95\% Cl] & $51.2(48.7-53.8)$ & $53.0(50.4-55.5)$ \\
\hline PPV $[95 \% \mathrm{Cl}]$ & $9.1(7.3-11.4)$ & $9.5(7.6-11.7)$ \\
\hline NPV $[95 \% \mathrm{Cl}]$ & $98.7(97.6-99.3)$ & $98.7(97.6-99.3)$ \\
\hline $\operatorname{LR}(+)[95 \% \mathrm{Cl}]$ & $1.80(1.64-1.98)$ & $1.87(1.70-2.06)$ \\
\hline $\operatorname{LR}(-)[95 \% \mathrm{Cl}]$ & $0.24(0.13-0.43)$ & $0.23(0.13-0.41)$ \\
\hline OR $[95 \% \mathrm{Cl}]$ & $7.56(3.87-14.77)$ & $8.12(4.16-15.86)$ \\
\hline $\mathrm{DA}[95 \% \mathrm{Cl}]$ & $53.2(50.7-55.6)$ & $54.8(52.4-57.3)$ \\
\hline \multicolumn{3}{|c|}{ Prehospital infection suspicion $(n=192$, sepsis $=54)$ : } \\
\hline Cut-off [points] & 2 & 2 \\
\hline Se \% $[95 \% \mathrm{Cl}]$ & $46.3(33.7-59.4)$ & $90.7(80.1-96.0)$ \\
\hline Sp \% $[95 \% \mathrm{Cl}]$ & $79.0(71.4-85.0)$ & $45.7(37.6-54.0)$ \\
\hline PPV $[95 \% \mathrm{Cl}]$ & $46.3(33.7-59.4)$ & $39.5(31.4-48.3)$ \\
\hline NPV $[95 \% \mathrm{Cl}]$ & $79.0(71.4-85.0)$ & $92.6(83.9-96.8)$ \\
\hline LR (+) $[95 \% \mathrm{Cl}]$ & $2.20(1.43-3.40)$ & $1.67(1.40-1.99)$ \\
\hline $\operatorname{LR}(-)[95 \% \mathrm{Cl}]$ & $0.68(0.51-0.90)$ & $0.20(0.09-0.48)$ \\
\hline OR $[95 \% \mathrm{Cl}]$ & $3.24(1.65-6.36)$ & $8.23(3.09-21.92)$ \\
\hline $\mathrm{DA}[95 \% \mathrm{Cl}]$ & $69.8(63.0-75.8)$ & $58.3(51.3-65.1)$ \\
\hline \multicolumn{3}{|c|}{ Prehospital non-infection suspicion $(n=1356$, sepsis $=28)$ : } \\
\hline Cut-off [points] & 1 & 3 \\
\hline Se \% $[95 \% \mathrm{Cl}]$ & $89.3(72.8-96.3)$ & $57.1(39.1-73.5)$ \\
\hline Sp \% $[95 \% \mathrm{Cl}]$ & $53.2(50.5-55.9)$ & $81.6(79.5-83.6)$ \\
\hline PPV $[95 \% \mathrm{Cl}]$ & $3.9(2.6-5.7)$ & $6.2(3.8-9.8)$ \\
\hline NPV $[95 \% \mathrm{Cl}]$ & $99.6(98.8-99.9)$ & $98.9(98.1-99.4)$ \\
\hline $\operatorname{LR}(+)[95 \% \mathrm{Cl}]$ & $1.91(1.96-2.20)$ & $3.11(2.21-4.37)$ \\
\hline $\operatorname{LR}(-)[95 \% \mathrm{Cl}]$ & $0.20(0.07-0.59)$ & $0.53(0.34-0.81)$ \\
\hline OR $[95 \% \mathrm{Cl}]$ & $9.49(2.85-31.58)$ & $5.92(2.77-12.68)$ \\
\hline $\mathrm{DA}[95 \% \mathrm{Cl}]$ & $54.0(51.3-56.6)$ & $81.1(79.0-83.1)$ \\
\hline
\end{tabular}

${ }^{\star}$ Bracketed numbers indicate 95\% confidence interval. qSOFA - quick Sequential Organ Failure Assessment, LqSOFA - Lactate quick Sequential Organ Failure Assessment, AUROC - area under receiver operating characteristic curve, Se - sensitivity, Sp - specificity, PPV - positive predictive value, NPV - negative predictive value, $L R$ - likelihood ratio, $O R$ - odds ratio, DA - diagnostic accuracy, $C l$ - confidence interval.

among these patients was 2 points. Precisely one of the advantages of this scale compared to qSOFA or other sepsis detection systems such as the systemic inflammatory response syndrome (SIRS) [28] is its excellent sensitivity. Sensitivity is crucial in serious cases whose early detection is so important and who rely on the most efficient management. Applying LqSOFA, few patients with sepsis would escape diagnosis if we used a 2-point cut-off point. Misses could happen if we only used the qSOFA scale, which is more evident among patients with suspected infection [6-8].

Therefore, the new LqSOFA scale represents a tool that professionals could implement and use 
F. Martín-Rodríguez, R. López-Izquierdo, M.A. Castro Villamor, C. delPozo-Vegas, I. Sánchez-Soberón, J.F. Delgado-Benito, V. Carbajosa Rodíguez, R. Leonardo, J.L. Martín-Conty

Table III. General sepsis score characteristics (statistics rates refer to sepsis diagnosis) between patients with suspicion of prehospital infection and non-infection suspicion

\begin{tabular}{|c|c|c|c|c|c|c|c|}
\hline \multirow[t]{2}{*}{ Parameter } & \multirow[t]{2}{*}{ Total } & \multicolumn{3}{|c|}{ Infection suspicion } & \multicolumn{3}{|c|}{ Non-infection suspicion } \\
\hline & & Non-sepsis & Sepsis & $P$-value & Non-sepsis & Sepsis & $P$-value \\
\hline \multicolumn{8}{|c|}{ qSOFA [points], $n(\%):$} \\
\hline$q S O F A=0$ & $761(49.2)$ & $44(86.3)$ & $7(13.7)$ & & 707 (99.6) & $3(0.4)$ & \\
\hline$q S O F A=1$ & $605(39.1)$ & $65(74.7)$ & $22(25.3)$ & & 502 (96.9) & $16(3.1)$ & \\
\hline qSOFA $=2$ & $158(10.2)$ & $26(61.9)$ & $16(38.1)$ & & $109(94.0)$ & $7(6.0)$ & \\
\hline qSOFA $=3$ & $24(1.6)$ & $3(25.0)$ & $9(75.0)$ & 0.001 & $10(83.3)$ & $2(16.7)$ & 0.001 \\
\hline \multicolumn{8}{|c|}{ LqSOFA [points], $n(\%):$} \\
\hline LqSOFA $=0$ & $279(18.0)$ & $16(100)$ & $0(0)$ & & $262(99.6)$ & $1(0.4)$ & \\
\hline LqSOFA $=1$ & $508(32.8)$ & $47(34.1)$ & $5(9.6)$ & & $452(99.1)$ & $4(0.9)$ & \\
\hline LqSOFA $=2$ & $437(28.2)$ & $43(71.7)$ & $17(28.3)$ & & $370(98.1)$ & $7(1.9)$ & \\
\hline LqSOFA $=3$ & $222(14.3)$ & $25(67.6)$ & $12(32.4)$ & & $175(94.6)$ & $10(5.4)$ & \\
\hline LqSOFA $=4$ & $84(5.4)$ & $6(33.4)$ & $12(66.7)$ & & $62(93.9)$ & $4(6.1)$ & \\
\hline LqSOFA $=5$ & $18(1.2)$ & $1(11.1)$ & $8(88.9)$ & 0.001 & $7(77.8)$ & $2(22.2)$ & 0.001 \\
\hline
\end{tabular}

*Values expressed as total number (fraction). qSOFA - quick Sequential Organ Failure Assessment, pLA - prehospital lactate, LqSOFA Lactate quick Sequential Organ Failure Assessment.

routinely in their clinical practice, on patients with suspected infection, but that does not improve the data provided by the qSOFA for the overall sample $[15,25,29,30]$.

In conclusion, sepsis should be considered a time-dependent disease by EMS, with specific activation and action codes, and identification should be the cornerstone on which to base the intervention. The use of the qSOFA and LqSOFA scales can help professionals suspect the early presence of sepsis and those patients at high risk.

The diagnostic performance of qSOFA and LqSOFA for the prediction of sepsis for any type of patient presented very good results. However, the LqSOFA has excellent sensitivity in patients with suspected infection, improving the predictive capacity of qSOFA.

These results performed at the prehospital level should be interpreted with caution, and should be verified with subsequent in-hospital confirmation.

This study has several limitations. In many cases, despite documented sepsis (clinically and analytically), the diagnosis is not explicitly stated in the patient's electronic medical record. One researcher from each hospital reviewed each record individually, but only those who were diagnosed by their medical team were counted as positive cases.

To guarantee internal validity, all personnel involved in the study received a procedure manual and initial training on how to collect data, measuring instruments and prehospital analytics. The MDs and ERNs of the EMS are professionals with documented experience, as are the staff of the EDs, whose technical skills and procedures are standardized. A fundamental issue is the loss of subjects; although it is impossible to plan the number of cases due to the unpredictability of the context, the distribution of daily entry of cases in the database was homogeneous.

The sample size was sufficient for the study, but it is necessary to conduct more powerful multicenter studies and in different geographical areas, in order to generalize the results of the LqSOFA scale.

\section{Acknowledgments}

Francisco Martín-Rodríguez ans Raúl López-Izquierdo - equal contribution.

The following research study has been developed in the Public Health System of the Community of Castilla y León (Spain). All professionals, ambulances and hospitals belong to the aforementioned public health system.

This research was funded by Gerencia Regional de Salud de Castilla y León (Spain), grant number GRS 1678/A/18 and Consejería de Sanidad de Castilla y León (Spain), grant number INT/E/02/19.

\section{Conflict of interest}

The authors declare no conflict of interest.

\section{References}

1. Singer M, Deutschman CS, Seymour CW, et al. The Third International Consensus Definitions for Sepsis and Septic Shock (Sepsis-3). JAMA 2016; 315: 801-10. 
2. Delahanty RJ, Alvarez J, Flynn LM, et al. Development and evaluation of a machine learning model for the early identification of patients at risk for sepsis. Ann Emerg Med 2019; 73: 334-44.

3. Smyth MA, Brace-McDonnell SJ, Perkins GD. Identification of adults with sepsis in the prehospital environment: a systematic review. BMJ Open 2016; 6: e01121.

4. Lane D, Ichelson RI, Drennan IR, et al. Prehospital management and identification of sepsis by emergency medical services: a systematic review. Emerg Med J 2016; 36: 408-13.

5. Alam N, Oskam E, Stassen PM, et al. Prehospital antibiotics in the ambulance for sepsis: a multicentre, open label, randomised trial. Lancet Respir Med 2018; 6: 40-50.

6. Kalantari A, Rezaie SR. Challenging the one-hour sepsis bundle. West J Emerg Med 2019; 20: 185-90.

7. Miyamoto K, Shibata N, Nakashima T, et al. Prehospital quick sequential organ failure assessment as a tool to predict in-hospital mortality. Am J Emerg Med 2018; 36: 1832-6.

8. Seymour CW, Liu VX, Iwashyna TJ, et al. Assessment of clinical criteria for sepsis: for the Third International Consensus Definitions for Sepsis and Septic Shock (Sepsis-3). JAMA 2016; 315: 762-74.

9. Morris E, McCartney D, Lasserson D, et al. Point-of-care lactate testing for sepsis at presentation to health care: a systematic review of patient outcomes. $\mathrm{Br} J$ Gen Pract 2017; 67: e859-70.

10. Swan KL, Avard BJ, Keene T. The relationship between elevated prehospital point-of-care lactate measurements, intensive care unit admission, and mortality: a retrospective review of adult patients. Aust Crit Care 2019; 32: 100-5.

11. Boland LL, Hokanson JS, Fernstrom KM, et al. Prehos pital lactate measurement by emergency medical services in patients meeting sepsis criteria. West J Emerg Med 2016; 17: 648-55.

12. Levy MM, Evans LE, Rhodes A. The surviving sepsis cam paign bundle: 2018 update. Intensive Care Med 2018; 44: 925-8.

13. Jones J, Lawner BJ. Prehospital sepsis care. Emerg Med Clin North Am 2017; 35: 175-83.

14. Smyth MA, Brace-McDonnell SJ, Perkins GD. Impact of prehospital care on outcomes in sepsis: a systematic review. West J Emerg Med 2016; 17: 427-37.

15. Sjösten O, Nilsson J, Herlitz J, et al. The prehospital assessment of patients with a final hospital diagnosis of sepsis: results of an observational study. Australas Emerg Care 2019; 22:187-92.

16. Johansson N, Spindler C, Valik J, et al. Developing a decision support system for patients with severe infection conditions in pre-hospital care. Int J Infect Dis 2018; 72: 40-8.

17. Filho RR, Rocha LL, Corrêa TD, et al. Blood lactate levels cutoff and mortality prediction in sepsis-time for a reappraisal? A retrospective cohort study. Shock 2016; 46: 480-5.

18. Gattinoni L, Vasques F, Camporota L, et al. Understanding lactatemia in human sepsis: potential impact for early management. Am J Respir Crit Care Med 2019; 200: 582-9.

19. Shetty AL, Thompson K, Byth K, et al. Serum lactate cutoffs as risk stratification tool for in-hospital adverse outcomes in emergency department patients screened for suspected sepsis. BMJ Open 2018; 8: e0154.

20. Bossuyt PM, Cohen JF, Gatsonis CA, et al. STARD 2015: updated reporting guidelines for all diagnostic accuracy studies. Ann Transl Med 2016; 4: 85.
21. Liu Z, Meng Z, Li Y, et al. Prognostic accuracy of the serum lactate level, the SOFA score and the qSOFA score for mortality among adults with Sepsis. Scand J Trauma Resusc Emerg Med 2019; 27: 51.

22 . Shetty $A$. Lactate $\geq 2 \mathrm{mmol} / \mathrm{L}$ plus qSOFA improves utility over qSOFA alone in emergency department patients presenting with suspected sepsis. Emerg Med Australas 2017; 29: 626-34.

23. Jung YT, Jeon J, Park JY, et al. Addition of lactic acid levels improves the accuracy of quick sequential organ failure assessment in predicting mortality in surgical patients with complicated intra-abdominal infections: a retrospective study. World J Emerg Surg 2018; 13: 14.

24. Song H, Moon HG, Kim SH. Efficacy of quick Sequential Organ Failure Assessment with lactate concentration for predicting mortality in patients with community-acquired pneumonia in the emergency department. Clin Exp Emerg Med 2019; 6: 1-8.

25. Ho KM, Lan NSH. Combining quick Sequential Organ Failure Assessment with plasma lactate concentration is comparable to standard Sequential Organ Failure Assessment score in predicting mortality of patients with and without suspected infection. J Crit Care 2017; 38: 1-5.

26. Shu E, Ives Tallman C, Frye W, et al. Pre-hospital qSOFA as a predictor of sepsis and mortality. Am J Emerg Med 2019; 37: 1273-8.

27. Dorsett $M$, Kroll M, Smith CS, et al. qSOFA has poor sensitivity for prehospital identification of severe sepsis and septic shock. Prehosp Emerg Care 2017; 21: 489-97.

28. Raith EP, Udy AA, Bailey M, et al. Prognostic accuracy of the SOFA score, SIRS criteria, and qSOFA score for in-hospital mortality among adults with suspected infection admitted to the intensive care unit. JAMA 2017; 317: 290-300

29. Singer AJ, Ng J, Thode HC, et al. Quick SOFA scores predict mortality in adult emergency department patients with and without suspected infection. Ann Emerg Med 2017; 69: 475-9.

30. Song JU, Sin CK, Park HK, et al. Performance of the quick Sequential (sepsis-related) Organ Failure Assessment score as a prognostic tool in infected patients outside the intensive care unit: a systematic review and metaanalysis. Crit Care 2018; 22: 28. 